\section{Targeting chemokines in fibrosis}

\section{By Tim Fulmer, Senior Writer}

A multinational team of researchers has shown that blocking chemokine signaling reduces liver fibrosis in mice. ${ }^{1}$ As additional proof of principle in models of advanced fibrosis, the next step may be testing chemokine antagonists that are already marketed or in trials for HIV and autoimmune disease.

A central step in hepatic fibrosis is the activation of hepatic stellate cells (HSCs) in response to chronic liver injury and their subsequent migration and production of fibrogenic extracellular matrix proteins (see Figure 1, "Targeting chemokines in liver disease")., ${ }^{2,3}$ Thus, blocking activation of HSCs should prevent or at least slow deposition of fibrotic tissue and help avoid liver failure.

Researchers from Columbia University, the University of California, San Diego and colleagues had previously focused on identifying proinflammatory chemokines that activated HSCs. They found that chemokine C-C motif ligand 5 (RANTES; CCL5) induced HSC proliferation and migration in vitro by binding to $\mathrm{CC}$ chemokine receptor 5 (CCR5; CD195) on the surface of HSCs. ${ }^{4}$

The new study, published in the Journal of Clinical Investigation, looked at whether a strategy targeting CCLs or their receptors on HSCs and other hepatic cells would have an antifibrotic effect in vivo.

In two mouse models of liver fibrosis, the chemokine ligands RANTES, macrophage inflammatory protein-1 $\alpha$ (Ccl3; Mip1a) and macrophage inflammatory protein- $1 \beta$ (Ccl4; Mip1b) were all significantly upregulated in the liver compared with in the livers of healthy controls $(p<0.05)$, as were Ccr5 and Ccr1 $(p<0.01)$.

In liver samples from hepatic cirrhosis patients, RANTES, CCR5 and CCR1 (CD191) mRNA levels were significantly higher than those in samples from healthy liver tissue.

To confirm the importance of the chemokine system in liver fibrosis, researchers treated one of the fibrosis models with a soluble, broad-spectrum inhibitor of CC chemokines. Compared with untreated littermates, treated mice showed significantly less fibrillar collagen in the liver and decreased levels of multiple profibrogenic markers ( $p<0.01$ for both).

With a handful of upregulated chemokines that could serve as potential antifibrotic targets, the team next turned to genetic knockout experiments to zero in on specific chemokines.

Mice with Ccr1 or Ccr5 knockout had significantly lower levels of fibrillar collagen in the liver than wild-type controls $(p<0.01)$. The livers of the knockout mice also had lower levels of profibrogenic markers and less proinflammatory macrophage infiltration than control livers.
Finally, the researchers confirmed that at least some of the antifibrotic effects of the chemokines were indeed mediated by HSCs.

Cultured HSCs isolated from the Ccrl and Ccr5 knockout mice had less capacity for migration in response to the ligands Rantes, Mipla and Mip1b than HSCs from wild-type mice. Migration of HSCs to areas of injury is a key component of the wound-healing response that can contribute to fibrosis over time.

\section{Establishing efficacy}

Taken together, the findings in the JCI article suggest that CCRs expressed on HSCs help mediate injury-induced hepatic fibrosis. Thus, antagonizing the CCRs or their ligands could help block activation of HSCs and prevent or slow disease progression.

"The JCI paper is definitive in establishing a mechanism whereby CCR1- and CCR5-mediated signaling in hepatic stellate cells and other liver cells contributes to fibrosis. Clearly a next step is to identify compounds that antagonize either or both of those receptors and thus block activation of hepatic stellate cells, directly or indirectly, to generate an antifibrotic effect," said Scott Friedman, professor of medicine and chief of the Division of Liver Diseases at the Mount Sinai School of Medicine.

A handful of CCR1 and CCR5 antagonists are either on the market or in the clinic. Pfizer Inc. markets the CCR5 antagonist Selzentry maraviroc to treat HIV infection, and at least five other companies have CCR5 antagonists in development to treat HIV.

CCX354, a CCR1 antagonist from ChemoCentryx Inc., is in Phase I testing to treat rheumatoid arthritis (RA). Ligand Pharmaceuticals Inc.'s CCR1 antagonist PS031291 is in preclinical development to treat multiple sclerosis (MS) and RA.

ChemoCentryx and Pfizer declined to comment on the findings in the JCI paper. Ligand did not reply to SciBX request for comment.

Other researchers wanted to see more extensive characterization of the CCR-targeting strategy in models of established fibrosis.

Shelia Violette, VP of research at Stromedix Inc., said it will be useful to see what happens when the antagonist is given long after the initial fibrogenic insult. That would help test whether targeting the receptors has a therapeutic effect on late-stage fibrotic disease, she noted.

Jeremy Duffield, assistant professor of medicine at Brigham and Women's Hospital and Harvard Medical School, agreed with Violette. "It will be key to look at the effects of CCR blockade on established fibrotic disease," he said. "That should help clarify whether the strategy is sufficient not only to block further progression of disease but also to promote resolution of disease and allow resorption of fibrotic tissue that already exists."

Duffield added that the work could be carried out using the same models that were described in the JCI paper.

Stromedix's STX-100, a humanized anti-integrin $\alpha_{v} \beta_{6} \mathrm{mAb}$ that was in-licensed from Biogen Idec Inc., is in preclinical development to treat lung, kidney and liver fibrosis. The company has completed a Phase I trial of STX-100 in healthy volunteers and is planning a Phase IIa trial in kidney transplant patients with chronic allograft dysfunction. 
Figure 1. Targeting chemokines in liver disease. A paper published in the Journal of Clinical Investigation makes a case for antagonizing chemokines or their receptors to prevent or halt progression of fibrosis associated with liver disease. A welldelineated series of events leads to hepatic fibrosis and ultimately to liver failure.

[a] Alcohol abuse, hepatitis infection and fatty liver resulting from metabolic syndrome can all trigger inflammation of the liver. Over time, this can lead to chronic liver injury.

[b] A key characteristic of the chronically injured liver is increased apoptosis and necrosis of hepatocytes, one of four cell types that make up the liver.

[c] Dying or dead hepatocytes release multiple factors that can trigger the activation of hepatic stellate cells (HSCs). Activated HSCs are the main cell type that drives hepatic fibrosis, and they do so by at least two different routes: recruitment of immune cells to areas of inflammation and injury, and secretion of fibrogenic extracellular matrix (ECM) proteins.

[d] Chronic activation of HSCs leads to excessive deposition of ECM proteins, which leads to fibrosis and scarring rather than healing and, if left unchecked, ultimately to liver failure and the need for a transplant.

The $\mathrm{JCl}$ paper suggests that blocking proinflammatory chemokine receptors CC chemokine receptor 1 (CCR1; CD191) and/or CCR5 (CD195) at steps $\mathbf{c}$ and $\mathbf{d}$ could slow or block excessive deposition of ECM proteins. A number of companies are pursuing other targets along this pathway for liver-related diseases. Selected compounds are displayed in the figure. Lead indications are listed for these compounds, which are not liver related in some cases.

By inhibiting integrin $\alpha_{\mathrm{v}} \beta_{6}$, STX-100 prevents activation of transforming growth factor- $\beta$ (TGFB; TGF $\beta$ ) and subsequent deposition of fibrogenic matrix proteins. ${ }^{5}$ Because the mAb likely acts downstream of the chemokine signaling process, STX-100 and CCR antagonists could potentially be complementary antifibrotic targeting strategies, Violette told SciBX.

Indeed, Friedman thinks a multipronged approach may ultimately be the way to go in liver fibrosis. "Hepatic fibrosis is a very complicated process involving multiple cell types and signaling pathways. Combination therapies that target a series of nodes in this process, from early-stage inflammation to late-stage established fibrosis, will likely yield the best therapeutic results," he said.

Robert Schwabe, one of two corresponding authors on the JCI paper and professor of medicine at Columbia, told SciBX he plans to test undisclosed CCR1 and CCR5 inhibitors in additional models of liver fibrosis.

The findings described in the JCI paper are not patented.

Fulmer, T. SciBX 2(27); doi:10.1038/scibx.2009.1062

Published online July 16, 2009

\section{REFERENCES}

1. Seki, E. et al. J. Clin. Invest.; published online June 15, 2009; doi:10.1172/JCl37444

Contact: Robert Schwabe, Columbia University, New York, N.Y. e-mail: rfs2102@columbia.edu

Contact: Ekihiro Seki, University of California, San Diego

School of Medicine, La Jolla, Calif. e-mail: ekseki@ucsd.edu

2. Friedman, S. Gastroenterology 134, 1655-1669 (2008)

3. Friedman, S. Physiol. Rev. 88, 125-172 (2008)

4. Schwabe, R. et al. Am. J. Physiol. Gastrointest. Liver Physiol. 285, G949-G958 (2003)

5. Patsenker, E. et al. Gastroenterology 135, 660-670 (2008)

\section{COMPANIES AND INSTITUTIONS MENTIONED}

Biogen Idec Inc. (NASDAQ:BIIB), Cambridge, Mass.

Brigham and Women's Hospital, Boston, Mass.

ChemoCentryx Inc., Mountain View, Calif.

Columbia University, New York, N.Y.

Harvard Medical School, Boston, Mass.

Ligand Pharmaceuticals Inc. (NASDAQ:LGND), San Diego, Calif.

Mount Sinai School of Medicine, New York, N.Y.

Pfizer Inc. (NYSE:PFE), New York, N.Y.

Stromedix Inc., Cambridge, Mass.

University of California, San Diego, La Jolla, Calif. 